\title{
DISTRIBUTION OF SCATTERING RESONANCES FOR GENERIC SCHRÖDINGER OPERATORS
}

\author{
TIEN-CUONG DINH AND VIÊT-ANH NGUYÊN
}

\begin{abstract}
Let $-\Delta+V$ be the Schrödinger operator acting on $L^{2}\left(\mathbb{R}^{d}, \mathbb{C}\right)$ with $d \geq 3$ odd. Here $V$ is a bounded real- or complex-valued function vanishing outside the closed ball of center 0 and radius $a$. If $V$ belongs to the class $\mathfrak{M}_{a}$ of potentials introduced by Christiansen, we show that when $r \rightarrow \infty$, the resonances of $-\Delta+V$, scaled down by the factor $r$, are asymptotically distributed, with respect to an explicit probability distribution on the closed lower unit half-disc of the complex plane. The rate of convergence is also considered for subclasses of potentials.
\end{abstract}

Classification AMS 2010: 35P25, 47A40.

Keywords: Schrödinger operator, resonance, scattering matrix, scattering pole.

\section{INTRODUCTION}

Let $\Delta$ denote the Laplacian operator on $\mathbb{R}^{d}$. In this work, we only consider $d$ odd because the case with $d$ even is of another nature. Let $V$ be a bounded complex-valued function with support in the closed ball $\overline{\mathbb{B}}_{a}$ of center 0 and radius $a$ in $\mathbb{R}^{d}$, that is, $V \in L^{\infty}\left(\overline{\mathbb{B}}_{a}, \mathbb{C}\right)$. The purpose of this work is to establish the distribution law for the resonances associated to the Schrödinger operator $-\Delta+V$ acting on $L^{2}\left(\mathbb{R}^{d}, \mathbb{C}\right)$ for "most of" potentials in $L^{\infty}\left(\overline{\mathbb{B}}_{a}, \mathbb{C}\right)$, or in $L^{\infty}\left(\overline{\mathbb{B}}_{a}, \mathbb{R}\right)$ if we only consider real potentials. The study of the asymptotic behavior of resonances has a long history and was intensively investigated during the last three decades. The reader can find in [2, 4, 10, 23, 27, 29, 34, 35] and the references therein an introduction to the subject.

Consider the complex parameter $\lambda \in \mathbb{C}$. If $\lambda$ is large enough with $\operatorname{Im}(\lambda)>0$, the operator $R_{V}(\lambda):=\left(-\Delta+V-\lambda^{2}\right)^{-1}$ on $L^{2}\left(\mathbb{R}^{d}, \mathbb{C}\right)$ is well-defined and is bounded. It depends holomorphically on $\lambda$. If $\chi$ is any smooth function with compact support such that $\chi V=V$, one can extend $\chi R_{V}(\lambda) \chi$ to a family of operators which depends meromorphically on $\lambda \in \mathbb{C}$. The poles of this family, which are called the resonances of the operator $-\Delta+V$, and their multiplicities do not depend on the choice of $\chi$. Let $\mathfrak{R}_{V}$ denote the set of resonances of $-\Delta+V$, where each element is counted according to its multiplicity. Denote by $n_{V}(r)$ the number of resonances of modulus $\leq r$ counted with multiplicity.

In dimension $d=1$, Zworski obtained in [31] the following estimate

$$
n_{V}(r)=\frac{4}{\pi} a r+o(r) \quad \text { as } \quad r \rightarrow \infty
$$

where $2 a$ is the diameter of the support of $V$, see also [11, 19, 22, 35]. In this paper we only consider the dimension $d \geq 3$.

Date: September 17, 2017. 
The upper bound for the number of resonances is well-understood while, in contrast, the lower bound is still not completely understood. Set

$$
N_{V}(r):=\int_{0}^{r} \frac{n_{V}(t)-n_{V}(0)}{t} d t .
$$

We have

$$
d N_{V}(r) \leq c_{d} a^{d} r^{d}+O\left(r^{d-1} \log r\right) \quad \text { as } \quad r \rightarrow \infty,
$$

where $c_{d}$ is defined in Section 2, This constant $c_{d}$ is sharp and was identified by Stefanov in [27]. The last estimate is a direct consequence of Propositions 3.1 and 3.2 below. It generalizes an estimate of Zworski in [33] where he obtained $o\left(r^{d}\right)$ instead of $O\left(r^{d-1} \log r\right)$. See also [24, 28] for more general results and [14, 15, 16] for earlier results.

Let $0<\nu \leq 1$ be a constant. The following family of potentials was introduced in [9]

$$
\mathfrak{M}_{a}^{\nu}:=\left\{V \in L^{\infty}\left(\overline{\mathbb{B}}_{a}, \mathbb{C}\right): n_{V}(r)-c_{d} a^{d} r^{d}=O\left(r^{d-\nu+\eta}\right) \text { as } r \rightarrow \infty \text { for every } \eta>0\right\} .
$$

Clearly, this is a subset of the following family introduced earlier by Christiansen in [5]

$$
\mathfrak{M}_{a}:=\left\{V \in L^{\infty}\left(\overline{\mathbb{B}}_{a}, \mathbb{C}\right): n_{V}(r)-c_{d} a^{d} r^{d}=o\left(r^{d}\right) \text { as } r \rightarrow \infty\right\} .
$$

We will call $\mathfrak{M}_{a}$ Christiansen class. By the results of [27, 32], $\mathfrak{M}_{a}$ contains all radial realvalued functions $V(z)=V(\|z\|)$ of class $\mathscr{C}^{2}$ on $\overline{\mathbb{B}}_{a}$ with $V(a) \neq 0$. In [3] Christiansen exhibits an example of a smooth complex-valued potential on $\overline{\mathbb{B}}_{a}$ which does not vanish on $b \mathbb{B}_{a}$ such that $\mathfrak{R}_{V}$ is empty. Such a function does not belong to $\mathfrak{M}_{a}$. Moreover, Vu and the first author proved in [9] that generic potentials in $L^{\infty}\left(\overline{\mathbb{B}}_{a}, \mathbb{C}\right)$ or in $L^{\infty}\left(\overline{\mathbb{B}}_{a}, \mathbb{R}\right)$ belong to $\mathfrak{M}_{a}^{3 / 16}$, see also [1, 6, 20, 21].

Consider a potential $V$ in $\mathfrak{M}_{a}$ and define for $r>0$

$$
\mu_{V, r}^{\Re}:=\frac{1}{c_{d} a^{d} r^{d}} \sum_{z \in \Re_{V}} \delta_{z / r},
$$

where $\delta_{z / r}$ denotes the Dirac mass at the point $z / r$. The fact that $V$ belongs to $\mathfrak{M}_{a}$ implies that the mass of $\mu_{V, r}^{\mathfrak{R}}$ on the unit disc tends to 1 as $r$ tends to infinity.

We will introduce later in Section 2 the positive constants $c_{d}, e_{d}$ and the function $h_{d}$ : $[0, \pi] \rightarrow \mathbb{R}_{+}$. Consider the measure $\mu^{0}$ with support in $\mathbb{R}$ which is absolutely continuous and has density $\frac{e_{d}}{2 \pi c_{d}}|x|^{d-1}$ with respect to the Lebesgue measure on $\mathbb{R}$. Consider also the measure $\mu^{-}$with support in the lower half-plane $\mathbb{C}_{-}$which is absolutely continuous with respect to the Lebesgue measure on $\mathbb{C}$ and has density

$$
\kappa(z):=\frac{1}{2 \pi c_{d}} r^{d-2}\left[d^{2} h_{d}(|\theta|)+h_{d}^{\prime \prime}(|\theta|)\right] \quad \text { with } \quad z=r e^{i \theta}, r=|z|, \theta \in(-\pi, 0) .
$$

We define $\mu_{\mathrm{MZ}}:=\mu^{0}+\mu^{-}$and call it Melrose-Zworski distribution. It will be shown later that $\mu_{\mathrm{MZ}}$ is a positive measure vanishing on the open upper half-plane $\mathbb{C}_{+}$. Moreover, its restriction to the unit disc is a probability measure. The Melrose-Zworski distribution is homogeneous of degree $d:$ if $A_{\lambda}: \mathbb{C} \rightarrow \mathbb{C}$ denotes the dilation $z \mapsto \lambda z$ with $\lambda>0$, then $\left(A_{\lambda}\right)^{*}\left(\mu_{\mathrm{MZ}}\right)=\lambda^{d} \mu_{\mathrm{MZ}}$.

For a set $W \subset \mathbb{C}$ and a number $r>0$, let $r W$ denote the dilation of $W$ by $r$, that is, $r W:=\{r z: z \in \Omega\}$. Let $n_{V, W}(r)$ be the number of resonances, counted with multiplicity, 
in $r W$. In particular, for $W=\mathbb{D}$ we have $n_{V, \mathbb{D}}(r)=n_{V}(r)$. Our first main result is the following theorem.

Theorem 1.1. Let $V$ be a function in the Christiansen class $\mathfrak{M}_{a}$. Then, the Schrödinger operator $-\Delta+V$ satisfies the Melrose-Zworski resonance law : when $r \rightarrow \infty$, the abovedefined measure $\mu_{V, r}^{\Re}$ converges weakly to the Melrose-Zworski distribution $\mu_{\mathrm{Mz}}$ on $\mathbb{C}$, i.e.

$$
\int \varphi d \mu_{V, r}^{\Re} \rightarrow \int \varphi d \mu_{\mathrm{MZ}} \text { as } \quad r \rightarrow \infty
$$

for all continuous functions $\varphi$ with compact support in $\mathbb{C}$. In particular, for every bounded open set $\Omega$ such that $b \Omega$ has zero area and $b \Omega \cap \mathbb{R}$ has zero length, we have

$$
n_{V, W}(r)=\mu_{\mathrm{MZ}}(\Omega) c_{d} a^{d} r^{d}+o\left(r^{d}\right) \quad \text { as } \quad r \rightarrow \infty
$$

for any set $W$ such that $\Omega \cap \mathbb{C}_{-} \subset W \subset \bar{\Omega}$, e.g. $W=\Omega \cap \mathbb{C}_{-}, \Omega \cap \overline{\mathbb{C}}_{-}, \Omega$ or $\bar{\Omega}$.

Consider now the following family of open sectors $\Omega\left(\theta_{1}, \theta_{2}\right)$ in the lower half-plane indexed by $0 \leq \theta_{1}<\theta_{2} \leq \pi$ :

$$
\Omega\left(\theta_{1}, \theta_{2}\right):=\left\{z \in \mathbb{D}: \theta_{1}-\pi<\arg z<\theta_{2}-\pi\right\} .
$$

We will applying Theorem 1.1 to these windows and obtain the following result, see [5, Prop. 1.1 and Cor. 1.4], see also [25, 26, 30] for related results.

Corollary 1.2 (Christiansen). For $\Omega:=\Omega\left(\theta_{1}, \theta_{2}\right)$, we have

$$
n_{V, \Omega}(r)=c\left(\theta_{1}, \theta_{2}\right) c_{d} a^{d} r^{d}+o\left(r^{d}\right) \quad \text { and } \quad n_{V, \bar{\Omega}}(r)=c\left(\theta_{1}, \theta_{2}\right) c_{d} a^{d} r^{d}+o\left(r^{d}\right) \quad \text { as } \quad r \rightarrow \infty \text {, }
$$

where

$$
c\left(\theta_{1}, \theta_{2}\right):=\frac{1}{2 \pi d c_{d}}\left[c\left(\theta_{2}\right)-c\left(\theta_{1}\right)+d^{2} \int_{\theta_{1}}^{\theta_{2}} h_{d}(\theta) d \theta\right] \quad \text { and } \quad c(\theta):= \begin{cases}h_{d}^{\prime}(\theta) & \text { for } 0<\theta<\pi \\ 0 & \text { for } \theta=0 \text { or } \pi .\end{cases}
$$

We can prove Theorem 1.1 using the last result and some standard techniques. However, we will consider in this paper a more direct and simpler approach. The novelties of our approach is that it not only gives us an explicit measure (Melrose-Zworski distribution), but also leads us to effective estimates of the rate of the convergence which will be presented in the next result.

Let $\Omega$ be any open set in $\mathbb{C}$ and let $\gamma>0$ be a positive number. Given $\mu$ and $\mu^{\prime}$ two positive measures on $\Omega$, define

$$
\operatorname{dist}_{\Omega, \gamma}\left(\mu, \mu^{\prime}\right):=\sup \left|\left\langle\mu-\mu^{\prime}, \varphi\right\rangle\right|,
$$

where the pairing $\left\langle\mu-\mu^{\prime}, \varphi\right\rangle$ denotes the integral of $\varphi$ with respect to the measure $\mu-\mu^{\prime}$ and the supremum is taken over all $\mathscr{C}^{\gamma}$ functions $\varphi$ with compact support in $\Omega$ with $\|\varphi\|_{\mathscr{C} \gamma} \leq 1$. As in [8], if $\gamma, \gamma^{\prime}$ and $\Omega, \Omega^{\prime}$ satisfy $0<\gamma \leq \gamma^{\prime}$ and $\Omega \Subset \Omega^{\prime} \subset \mathbb{C}$, on any subset of measures whose masses on $\Omega^{\prime}$ are bounded by a constant, we have the following inequalities for some constant $c>0$

$$
\operatorname{dist}_{\Omega, \gamma^{\prime}} \leq \operatorname{dist}_{\Omega, \gamma} \leq c\left[\operatorname{dist}_{\Omega^{\prime}, \gamma^{\prime}}\right]^{\gamma / \gamma^{\prime}} .
$$

The function $\operatorname{dist}_{\Omega, \gamma}$ is a semi-distance on the space of locally finite positive measures on $\mathbb{C}$. Note that $\operatorname{dist}_{\Omega, 1}$ is related to the well-known Kantorovich-Wasserstein distance for measures. 
Theorem 1.3. Let $V$ be a function in the class $\mathfrak{M}_{a}^{\nu}$ for some $0<\nu \leq 1$, let $\eta>0$ be an arbitrary constant and let $\Omega$ be a bounded domain in $\mathbb{C}$. Then for every number $0<\gamma \leq 1$, we have that

$$
\operatorname{dist}_{\Omega, \gamma}\left(\mu_{V, r}^{\Re}, \mu_{\mathrm{MZ}}\right) \leq c r^{-\gamma \nu / 2+\eta} \text { for } r \text { large enough, }
$$

where $c>0$ is a constant which depends on $a, V, \gamma, \nu$ and $\eta$ but is independent of $r$. Moreover, if the boundary of $\Omega$ is piecewise smooth and transverse to the real line $\mathbb{R}$, then

$$
n_{V, W}(r)=\mu_{\mathrm{MZ}}(\Omega) c_{d} a^{d} r^{d}+o\left(r^{d-\nu / 3+\eta}\right) \quad \text { as } \quad r \rightarrow \infty
$$

for any set $W$ such that $\Omega \cap \mathbb{C}_{-} \subset W \subset \bar{\Omega}$, e.g. $W=\Omega \cap \mathbb{C}_{-}, \Omega \cap \overline{\mathbb{C}}_{-}, \Omega$ or $\bar{\Omega}$.

Notation and convention. Denote by $\mathbb{B}_{a}$ the open ball of center 0 and radius $a$ in $\mathbb{R}^{d}$. For a set $\Omega \subset \mathbb{C}$ and $r>0$, let $r \Omega:=\{r z: z \in \Omega\}$ and let $b \Omega$ denote the boundary of $\Omega$. Let $\mathbb{D}$ (resp. $\mathbb{D}(s)$ ) be the open unit disc (resp. the disc of center 0 and radius $s$ ) in $\mathbb{C}$. Define $\mathbb{C}_{ \pm}:=\{z \in \mathbb{C}: \pm \operatorname{Im} z>0\}$ and $\mathbb{D}_{ \pm}:=\mathbb{D} \cap \mathbb{C}_{ \pm}$. The function $h_{d}$ and the constant $c_{d}$ are introduced in Section 2. Write $\arg z:=\theta$ and $\log z:=\log r+i \theta$ for $z=r e^{i \theta}$ with $r>0$ and $\theta \in[-\pi, \pi]$. Recall that $d^{c}:=\frac{1}{2 \pi i}(\partial-\bar{\partial})$ and $d d^{c}=\frac{i}{\pi} \partial \bar{\partial}$. Both Leb and $d x d y$ (resp. Leb $\mathbb{R}_{\mathbb{R}}$ and $d x$ ) denote the Lebesgue measure on $\mathbb{C}$ (resp. on $\mathbb{R}$ ), where we use the canonical coordinates $z=x+i y$. Let $L^{\infty}\left(\overline{\mathbb{B}}_{a}, \mathbb{C}\right)$ (resp. $L^{\infty}\left(\overline{\mathbb{B}}_{a}, \mathbb{R}\right)$ ) be the space of all bounded complex- (resp. real-) valued functions with support in $\overline{\mathbb{B}}_{a}$. The constants we use can be changed from line to line but they are independent of $r$. The notations $\lesssim$ and $\gtrsim$ mean inequalities up to a multiplicative constant.

Acknowledgement. The first author was supported by Start-Up Grant R-146-000-204133 from the National University of Singapore. The paper was partially prepared during the visit of the first author at the Freie Universität Berlin and of the second author at the National University of Singapore. They would like to thank these organizations, the Alexander von Humboldt foundation and Hélène Esnault for their hospitality and support.

\section{Properties of SOME POSITIVE MEASURES}

In this section, we will give basic results on positive measures in $\mathbb{C}$ and their potentials, see [7, 13, 18]. The measures we consider are locally finite Borel measures. We also study some properties of the Melrose-Zworski distribution that will be used later in the proof of our main results.

Lemma 2.1. Let $\mu_{k}$, with $k \in \mathbb{N}$, and $\mu$ be positive measures in $\mathbb{C}$ such that $\mu_{k}$ converges to $\mu$ weakly as $k$ tends to $\infty$. Let $\Omega$ be a bounded opens set in $\mathbb{C}$ and assume that $\mu(b \Omega)=0$. Then $\mu_{k}(W) \rightarrow \mu(\Omega)$ as $k \rightarrow \infty$ for every set $W$ such that $\Omega \subset W \subset \bar{\Omega}$.

Proof. It is enough to prove that $\mu_{k}(\Omega) \rightarrow \mu(\bar{\Omega})$ and $\mu_{k}(\bar{\Omega}) \rightarrow \mu(\Omega)$. Choose two sequences of continuous functions $0 \leq \chi_{n} \leq \rho_{n} \leq 1$ with compact support in $\mathbb{C}$ such that $\chi_{n}$ increases to the characteristic function of $\Omega$ and $\rho_{n}$ decreases to the characteristic function of $\bar{\Omega}$. We have for each $n$

$$
\liminf _{k \rightarrow \infty} \mu_{k}(\Omega) \geq \liminf _{k \rightarrow \infty}\left\langle\mu_{k}, \chi_{n}\right\rangle=\left\langle\mu, \chi_{n}\right\rangle .
$$

Taking $n \rightarrow \infty$ gives

$$
\liminf _{k \rightarrow \infty} \mu_{k}(\Omega) \geq \mu(\Omega)=\mu(\bar{\Omega})
$$


We use here that $\mu(b \Omega)=0$. Similarly, we have

Taking $n \rightarrow \infty$ gives

$$
\limsup _{k \rightarrow \infty} \mu_{k}(\bar{\Omega}) \leq \limsup _{k \rightarrow \infty}\left\langle\mu_{k}, \rho_{n}\right\rangle=\left\langle\mu, \rho_{n}\right\rangle .
$$

$$
\limsup _{k \rightarrow \infty} \mu_{k}(\bar{\Omega}) \leq \mu(\bar{\Omega})=\mu(\Omega) .
$$

Therefore, we get $\mu_{k}(\Omega) \rightarrow \mu(\Omega)$ and $\mu_{k}(\bar{\Omega}) \rightarrow \mu(\Omega)$. This implies the lemma.

We will give now some results which allow us to get the rate of convergence of positive measures on $\mathbb{C}$. Let $\Omega$ be an open set in $\mathbb{C}$, not necessarily bounded, such that $b \Omega$ is compact. For $\epsilon>0$, denote by $(b \Omega)_{\epsilon}$ the set of points $z$ of distance less than $\epsilon$ to $b \Omega$. Let $\vartheta_{\Omega}(\epsilon)$ be the infimum of the numbers $\vartheta>0$ such that

- there is a function $0 \leq \chi \leq 1$ of class $\mathscr{C}^{2}$ with support in $\Omega$ and equal to 1 on $\Omega \backslash(b \Omega)_{\epsilon}$ such that $\|\chi\|_{\mathscr{C}^{2}} \leq \vartheta$;

- there is a function $0 \leq \rho \leq 1$ of class $\mathscr{C}^{2}$ with support in $\Omega \cup(b \Omega)_{\epsilon}$ and equal to 1 on $\bar{\Omega}$ such that $\|\rho\|_{\mathscr{C}^{2}} \leq \vartheta$.

If such functions do not exist, we define $\vartheta_{\Omega}(\epsilon):=+\infty$.

Lemma 2.2. Let $u_{1}$ and $u_{2}$ be two subharmonic functions on an open set $U$ in $\mathbb{C}$. Define $\mu_{1}:=d d^{c} u_{1}$ and $\mu_{2}:=d d^{c} u_{2}$. Let $\Omega$ be an open set in $\mathbb{C}$ such that $\Omega \Subset U$ and let $0<\gamma \leq 2$ be a constant. Then there is a constant $c>0$ depending only on $\Omega, U, \gamma$ such that

$\operatorname{dist}_{\Omega, \gamma}\left(\mu_{1}, \mu_{2}\right) \leq c\left\|u_{1}-u_{2}\right\|_{L^{1}(U)}^{\gamma / 2} \quad$ and $\quad\left|\mu_{1}(\Omega)-\mu_{2}(\Omega)\right| \leq \mu_{1}\left((b \Omega)_{\epsilon}\right)+c \vartheta_{\Omega}(\epsilon)\left\|u_{1}-u_{2}\right\|_{L^{1}(U)}$ for $\epsilon>0$ small enough. Moreover, the last estimate still holds if we replace $\mu_{1}(\Omega)-\mu_{2}(\Omega)$ by $\mu_{1}(\bar{\Omega})-\mu_{2}(\bar{\Omega})$.

Proof. We first prove the first inequality for $\gamma=2$. Let $\varphi$ be any $\mathscr{C}^{2}$ function with compact support in $\Omega$ such that $\|\varphi\|_{\mathscr{C}^{2}} \leq 1$. By Stokes formula, we have

$$
\left|\left\langle\mu_{1}-\mu_{2}, \varphi\right\rangle\right|=\left|\left\langle d d^{c}\left(u_{1}-u_{2}\right), \varphi\right\rangle\right|=\left|\left\langle u_{1}-u_{2}, d d^{c} \varphi\right\rangle\right|=\left|\int_{\Omega}\left(u_{1}-u_{2}\right) d d^{c} \varphi\right| .
$$

Since $d d^{c} \varphi$ is a bounded differential form, the last integral is bounded by a constant times $\left\|u_{1}-u_{2}\right\|_{L^{1}(U)}$. By (1.4), the first estimate in the lemma holds for $\gamma=2$. If $\Omega^{\prime}$ is an open set such that $\Omega \Subset \Omega^{\prime} \Subset U$, we obtain in the same way that $\operatorname{dist}_{\Omega^{\prime}, 2}\left(\mu_{1}, \mu_{2}\right)$ is bounded by a constant times $\left\|u_{1}-u_{2}\right\|_{L^{1}(U)}$. This, together with (1.5), imply the first assertion in the lemma for every $0<\gamma \leq 2$.

Fix an arbitrary constant $\vartheta>\vartheta_{\Omega}(\epsilon)$ for $\epsilon>0$ small enough. Let $\chi$ and $\rho$ be as above. We have

$$
\mu_{1}(\Omega)-\mu_{2}(\Omega) \leq \mu_{1}(\Omega)-\left\langle\mu_{2}, \chi\right\rangle \leq \mu_{1}\left((b \Omega)_{\epsilon}\right)+\left\langle\mu_{1}, \chi\right\rangle-\left\langle\mu_{2}, \chi\right\rangle .
$$

Recall that $\|\chi\|_{\mathscr{C}^{2}} \leq \vartheta$. As it was done for $\varphi$ above, we obtain that $\left\langle\mu_{1}, \chi\right\rangle-\left\langle\mu_{2}, \chi\right\rangle$ is bounded from above by a constant times $\vartheta\left\|u_{1}-u_{2}\right\|_{L^{1}(U)}$. Therefore, we have for some constant $c>0$

$$
\mu_{1}(\Omega)-\mu_{2}(\Omega) \leq \mu_{1}\left((b \Omega)_{\epsilon}\right)+c \vartheta\left\|u_{1}-u_{2}\right\|_{L^{1}(U)} .
$$

Similarly, using the function $\rho$, we get

$$
\mu_{1}(\Omega)-\mu_{2}(\Omega) \geq-\mu_{1}\left((b \Omega)_{\epsilon}\right)-c \vartheta\left\|u_{1}-u_{2}\right\|_{L^{1}(U)} .
$$

This implies the second estimate in the lemma. Note that the same proof holds when we replace $\Omega$ by $\bar{\Omega}$. 
Later, we will use the last lemma for $\Omega$ a bounded open set with piecewise smooth boundary. The following result is then useful. We say that an open set $\Omega$ with compact boundary is nice if $\vartheta_{\Omega}(\epsilon)=O\left(\epsilon^{-2}\right)$ as $\epsilon$ tends to 0 .

Lemma 2.3. Let $\Omega$ be a bounded simply connected open set in $\mathbb{C}$ with piecewise smooth boundary. Assume that the angle at each singular point of its boundary is strictly smaller than $\pi$. Then $\Omega$ is nice.

Proof. Consider first the case of smooth boundary. We can find a defining smooth function $\tau: \mathbb{C} \rightarrow \mathbb{R}$ such that $\Omega:=\{\tau<0\}$ and that $d \tau \neq 0$ on $b \Omega$. Fix a smooth function $\chi_{0}: \mathbb{R} \rightarrow[0,1]$ such that $\chi_{0}=1$ on $(-\infty, 0]$ and $\chi_{0}=0$ on $[1 / 2, \infty)$. Fix also two constants $\epsilon_{0}$ small enough and $A>0$ large enough. For every $0<\epsilon \leq \epsilon_{0}$, we can check that the functions

$$
\chi(z):=\chi_{0}\left(A \epsilon^{-1} \tau(z)+1\right) \quad \text { and } \quad \rho(z):=\chi_{0}\left(A \epsilon^{-1} \tau(z)-1\right)
$$

satisfy the conditions required in the definition of $\vartheta_{\Omega}(\epsilon)$. Their $\mathscr{C}^{2}$ norms are bounded by a constant times $\epsilon^{-2}$. This implies the result for the smooth case. Note that the $\mathscr{C}^{1}$ norms of $\chi$ and $\rho$ are bounded by a constant times $\epsilon^{-1}$.

Consider the general case. Observe that we can find a finite number of simply connected bounded open sets $\Omega_{1}, \ldots, \Omega_{k}$ with smooth boundaries whose intersection is equal to $\Omega$. Moreover,

(1) Each smooth piece of $b \Omega$ is contained in $b \Omega_{i}$ for exactly one index $i$ and conversely, for each $i, b \Omega_{i}$ contains exactly one smooth piece of $b \Omega$;

(2) For $i \neq j, b \Omega_{i}, b \Omega_{j}$ intersect at exactly 2 points and the intersection is transversal.

(3) For all distinct indexes $i, j, l$, the intersection of $b \Omega_{i}, b \Omega_{j}$ and $b \Omega_{l}$ is empty.

To see this point, we can use a smooth diffeomorphism of $\mathbb{C}$ in order to reduce the problem to the case of a convex polygon.

Fix a constant $c>0$ small enough. We only need to consider $\epsilon>0$ small enough and define $\epsilon^{\prime}:=c \epsilon$. For each $j=1, \ldots, k$, we can choose the functions $\chi_{j}$ and $\rho_{j}$ as in the definition of $\vartheta_{\Omega_{j}}\left(\epsilon^{\prime}\right)$ associated with $\Omega_{j}, \epsilon^{\prime}$ instead of $\Omega, \epsilon$ and such that $\left\|\chi_{j}\right\|_{\mathscr{C}^{2}}=O\left(\epsilon^{-2}\right)$ and $\left\|\chi_{j}\right\|_{\mathscr{C}^{1}}=O\left(\epsilon^{-1}\right)$. Set $\chi:=\chi_{1} \ldots \chi_{k}$ and $\rho:=\rho_{1} \ldots \rho_{k}$. We can check that these functions satisfy the conditions required in the definition of $\vartheta_{\Omega}(\epsilon)$ as in the case where $\Omega$ is a convex polygon. Moreover, both $\|\chi\|_{\mathscr{C}^{2}}$ and $\|\rho\|_{\mathscr{C}^{2}}$ are bounded by a constant times $\epsilon^{-2}$. So $\Omega$ is a nice open set.

We will also need the following auxiliary results. Consider a domain $\Omega$ in $\mathbb{C}$ which is symmetric with respect to the real line $\mathbb{R}$. Define $\Omega_{+}:=\Omega \cap \mathbb{C}_{+}, \Omega_{-}:=\Omega \cap \mathbb{C}_{-}$and $L:=\Omega \cap \mathbb{R}$.

Lemma 2.4. Let $u$ be a subharmonic function on $\Omega_{+}$such that $u \geq 0$ and $u(z)$ tends to 0 when $z$ tends to $L$. Then the function

$$
u_{0}(z):= \begin{cases}u(z) & \text { for } z \in \Omega_{+} \\ 0 & \text { for } z \in \Omega_{-} \cup L .\end{cases}
$$

is subharmonic on $\Omega$. 
Proof. Consider for $\epsilon>0$ the function

$$
u_{\epsilon}(z):= \begin{cases}\max (u(z), \epsilon) & \text { for } z \in \Omega_{+} \\ \epsilon & \text { for } z \in \Omega_{-} \cup L .\end{cases}
$$

Clearly, this function is subharmonic in $\Omega_{+} \cup \Omega_{-}$because the maximum of two subharmonic functions is subharmonic. Since subharmonic functions are upper semi-continuous, $u_{\epsilon}=\epsilon$ in a neighbourhood of $L$. So it is also subharmonic in a neighbourhood of $L$. It follows that $u_{\epsilon}$ is subharmonic on $\Omega$. When $\epsilon$ decreases to $0, u_{\epsilon}$ decreases to $u_{0}$. Therefore, $u_{0}$ is also subharmonic.

Lemma 2.5. Let $u$ be a continuous function on $\Omega_{+} \cup L$ such that $\frac{\partial u}{\partial y}$ exists and is continuous there. Assume that $u$ is subharmonic on $\Omega^{+}$. Define the function $\tilde{u}: \Omega \rightarrow \mathbb{R}$ by

$$
\tilde{u}(z):= \begin{cases}u(z) & \text { for } z \in \Omega_{+} \cup L \\ u(\bar{z}) & \text { for } z \in \Omega_{-} .\end{cases}
$$

(1) The function $\tilde{u}$ is subharmonic in $\Omega$ if and only if $\frac{\partial u}{\partial y}(z) \geq 0$ for all $z \in L$.

(2) We have that $d d^{c} \tilde{u}$ is a measure on $\Omega$. Moreover, its restriction to $L$ is absolutely continuous and has density $\frac{1}{\pi} \frac{\partial u}{\partial y}(x)$ with respect to the Lebesgue measure on $L$.

In particular, we have $d d^{c}|y|=\frac{1}{\pi} \operatorname{Leb}_{\mathbb{R}}$ on $\mathbb{C}$.

Proof. (1) Assume that $\tilde{u}$ is subharmonic. We show that $\frac{\partial u}{\partial y}(z) \geq 0$ for all $z \in L$. Assume by contradiction that $\frac{\partial u}{\partial y}(a)<0$ at some point $a \in L$. For simplicity, we can suppose $a=0$. Let $\rho \geq 0$ be a smooth function on $\mathbb{R}$ with compact support and with integral 1 . Consider the following functions obtaining by a convolution with $\rho^{\epsilon}(t):=\epsilon^{-1} \rho\left(\epsilon^{-1} t\right)$ :

$$
u^{\epsilon}(z):=\left(\rho^{\epsilon} * u\right)(z):=\int_{\mathbb{R}} u(z+t) \rho^{\epsilon}(t) d t
$$

and

$$
\tilde{u}^{\epsilon}(z):=\left(\rho^{\epsilon} * \tilde{u}\right)(z):=\int_{\mathbb{R}} \tilde{u}(z+t) \rho^{\epsilon}(t) d t .
$$

For $\epsilon$ small enough, these functions satisfy similar properties as $u$ and $\tilde{u}$ do. In particular, $\tilde{u}^{\epsilon}$ is subharmonic near 0 and $\frac{\partial u^{\epsilon}}{\partial y}(0)<0$ because

$$
\frac{\partial u^{\epsilon}}{\partial y}(z)=\left(\rho^{\epsilon} * \frac{\partial u}{\partial y}\right)(z)=\int_{\mathbb{R}} \frac{\partial u}{\partial y}(z+t) \rho^{\epsilon}(t) d t .
$$

Moreover, the restrictions of $u^{\epsilon}$ and $\tilde{u}^{\epsilon}$ to $\mathbb{R}$ are smooth. So we can replace $u, \tilde{u}$ by $u^{\epsilon}, \tilde{u}^{\epsilon}$ in order to assume that $u$ is smooth on $L$.

Adding to $\tilde{u}$ a suitable (harmonic) affine function in $x$ allows us to assume that $u(0)=0$ and $\frac{\partial u}{\partial x}(0)=0$. Fix small enough constants $\delta>0$ and $r>0$ such that $\frac{\partial u}{\partial y}(z) \leq-2 \delta$ for $z \in \Omega_{+} \cup L$ with $|z| \leq r$ and $u(z) \leq \delta|z|$ for $z \in[-r, r]$. For $\theta \in[0, \pi]$, we have using the above property of $\frac{\partial u}{\partial y}(z)$ that

$$
u\left(r e^{i \theta}\right)-u(r \cos \theta) \leq-2 \delta\left|r e^{i \theta}-r \cos \theta\right|=-2 \delta r \sin \theta
$$

hence

$$
u\left(r e^{i \theta}\right) \leq u(r \cos \theta)-2 \delta r \sin \theta \leq \delta r(|\cos \theta|-2 \sin \theta) .
$$

So we have for $\theta \in[-\pi, \pi]$

$$
\tilde{u}\left(r e^{i \theta}\right) \leq \delta r(|\cos \theta|-2|\sin \theta|) .
$$


This contradicts the following submean inequality for subharmonic functions

$$
\tilde{u}(0) \leq \frac{1}{2 \pi} \int_{-\pi}^{\pi} \tilde{u}\left(r e^{i \theta}\right) d \theta
$$

because $\tilde{u}(0)=0$. Thus, $\frac{\partial u}{\partial y}(z) \geq 0$ for $z \in L$.

Assume now that $\frac{\partial u}{\partial y}(z) \geq 0$ for $z \in L$. We have to show that $\tilde{u}$ is subharmonic. Recall that if a sequence of subharmonic functions converges locally uniformly, then the limit is also a subharmonic function. Therefore, we can replace $u$ by $u+\epsilon y$ for $\epsilon>0$ in order to assume that $\frac{\partial u}{\partial y}(z)>0$ for $z \in L$. By continuity, the last inequality holds for $z$ in a neighbourhood of $L$.

The problem is local near the points of of $L$. So without loss of generality, we can assume that $\Omega$ is the square $(-1,1) \times(-1,1)$ and that $\frac{\partial u}{\partial y}(z)>0$ on $\Omega_{+} \cup L$. It follows that the restriction of $u$ to $\{x\} \times[0,1)$ is strictly increasing for every $x \in(-1,1)$. For $0<\eta<1 / 2$, consider the functions

$$
v_{\eta}(z):=u(2 \eta i+\bar{z}) \quad \text { and } \quad \tilde{u}_{\eta}(z):= \begin{cases}u(z) & \text { when } \eta \leq y<1 \\ v_{\eta}(z) & \text { when }-1+2 \eta<y \leq \eta .\end{cases}
$$

Using that $u$ is increasing in vertical lines, we deduce that

$$
\tilde{u}_{\eta}(z):= \begin{cases}u(z) & \text { when } \eta<y<1 \\ \max \left(u(z), v_{\eta}(z)\right) & \text { when } 0<y<2 \eta \\ v_{\eta}(z) & \text { when }-1+2 \eta<y<\eta .\end{cases}
$$

Observe that $v_{\eta}$ is subharmonic on $(-1,1) \times(-1+2 \eta, 2 \eta)$. Therefore, the last formula for $\tilde{u}_{\eta}$ implies that this function is subharmonic everywhere in $(-1,1) \times(1-2 \eta, 1)$. We use that the maximum of two subharmonic functions is subharmonic. Finally, when $\eta \rightarrow 0$ we see that $\tilde{u}_{\eta} \rightarrow \tilde{u}$ locally uniformly on $(-1,1) \times(-1,1)$. It follows that $\tilde{u}$ is subharmonic in $(-1,1) \times(-1,1)$.

(2) Recall that $d d^{c} \tilde{u}$ is a positive measure on $\Omega_{+} \cup \Omega_{-}$. So the problem is local in a neighbourhood of each point of $L$. We can assume as above that $\Omega$ is the square $(-1,1) \times(-1,1)$ and that the function $m(x):=\frac{1}{\pi} \frac{\partial u}{\partial y}(x)$ is uniformly continuous and bounded on $L=(-1,1)$. We extend $m(x)$ to a function on $\mathbb{R}$ which vanishes outside $L$. Define $\nu:=m(x) d x$ which is a finite measure on $\mathbb{C}$ with support in $\bar{L}$. We first show that $d d^{c} \tilde{u} \geq \nu$. This implies that $d d^{c} \tilde{u}$ is a measure on $\Omega$.

Consider the logarithmic potential of the measure $\nu$ defined by

$$
v(z):=\int_{\mathbb{R}} \log |z-t| m(t) d t \quad \text { for } z \in \mathbb{C} .
$$

It satisfies $v(\bar{z})=v(z), d d^{c} v=\nu$ and therefore, $v$ is harmonic on $\mathbb{C} \backslash \bar{L}$. It is not difficult to show that this function is continuous in $\mathbb{C}$. Moreover, for $z=x+i y$ with $y>0, x^{\prime}:=y^{-1} x$ and $t^{\prime}:=y^{-1}(t-x)$, we have

$$
\frac{\partial v}{\partial y}(z)=\frac{\partial}{\partial y} \int_{\mathbb{R}} \frac{1}{2} \log \left((x-t)^{2}+y^{2}\right) m(t) d t=\int_{\mathbb{R}} \frac{y}{(x-t)^{2}+y^{2}} m(t) d t=\int_{\mathbb{R}} \frac{m\left(x+y t^{\prime}\right)}{t^{\prime 2}+1} d t^{\prime} .
$$

Recall that the integral of $\left(t^{\prime 2}+1\right)^{-1}$ on $\mathbb{R}$ is $\pi$. Thus, it is not difficult to see from the last computation that the function $\frac{\partial v}{\partial y}(z)$ on $\Omega_{+}$extends to a continuous function on $\Omega_{+} \cup L$. 
It converges uniformly to $\pi m(x)$ when $y \rightarrow 0$. We conclude that $\frac{\partial v}{\partial y}(z)$ exists and is continuous on $\Omega_{+} \cup L$ and equal to $\frac{\partial u}{\partial y}(z)$ on $L$.

Since the function $v$ is harmonic on $\Omega_{+}$, the function $u-v$ is subharmonic on $\Omega_{+}$. We can apply the first assertion (1) to $u-v, \tilde{u}-v$ instead of $u, \tilde{u}$ and deduce that $\tilde{u}-v$ is subharmonic. It follows that $d d^{c} \tilde{u} \geq d d^{c} v=\nu$. For the rest of the proof, without loss of generality, we can replace $u, \tilde{u}$ by $u-v, \tilde{u}-v$ in order to assume that $\frac{\partial u}{\partial y}(z)=0$ on $L$ and we still need to check that $d d^{c} \tilde{u}$ has no mass on $L$.

Assume by contradiction that $d d^{c} \tilde{u}$ doesn't vanish on $L$. Replacing $u, \tilde{u}$ by $u^{\epsilon}, \tilde{u}^{\epsilon}$ which are defined at the beginning of the proof, we can assume that the restriction of $d d^{c} \tilde{u}$ to $L$ is a positive measure absolutely continuous with continuous and bounded density with respect to the Lebesgue measure on $L$. Denote this measure by $\nu^{\prime}=m^{\prime}(x) d x$ and $v^{\prime}(z)$ its logarithmic potential. Since $d d^{c} \tilde{u} \geq \nu^{\prime}$, the function $\tilde{u}-v^{\prime}$ is subharmonic in $\Omega$. A computation as before shows that

$$
\frac{\partial\left(u-v^{\prime}\right)}{\partial y}(z)=-\frac{\partial v^{\prime}}{\partial y}(z)=-\pi m^{\prime}(z) \quad \text { for } \quad z \in L
$$

This contradicts the assertion (1) applied to $u-v^{\prime}$ and $\tilde{u}-v^{\prime}$ which implies that the last partial derivative of $u-v^{\prime}$ should be non-negative on $L$. This ends the proof of the lemma.

Now, we recall some notions and results related to the Melrose-Zworski distribution. Let $\rho$ be the continuous function on $\overline{\mathbb{C}}_{+} \backslash\{0\}$ defined by

$$
\rho(z):=\log \frac{1+\sqrt{1-z^{2}}}{z}-\sqrt{1-z^{2}}
$$

which extends the real-valued function in $z \in(0,1)$ given by the same formula. Set

$$
h_{d}(\theta):=\frac{4}{(d-2) !} \int_{0}^{\infty} \frac{\max \left(-\operatorname{Re} \rho\left(t e^{i \theta}\right), 0\right)}{t^{d+1}} d t \quad \text { for } 0<\theta<\pi,
$$

and set $h_{d}(0):=0$ and $h_{d}(\pi):=0$. It can be shown that $h_{d}$ is continuous on $[0, \pi]$ and is a real analytic function on $(0, \pi)$. The constant $c_{d}$ appearing in the Introduction is given by

$$
c_{d}:=\frac{d}{2 \pi} \int_{0}^{\pi} h_{d}(\theta) d \theta=\frac{2 d}{\pi(d-2) !} \int_{\operatorname{Im} z>0} \frac{\max (-\operatorname{Re} \rho(z), 0)}{|z|^{d+2}} d x d y .
$$

We infer from (2.1) and (2.2) that $\operatorname{Re} \rho(z)$ is invariant under the map $z \mapsto-\bar{z}$ and hence

$$
h_{d}(\pi / 2+\theta)=h_{d}(\pi / 2-\theta) \text { for } 0 \leq \theta \leq \pi / 2 .
$$

Using the gamma function $\Gamma$ consider also the constant

$$
e_{d}:=\sqrt{\pi} \frac{\Gamma\left(\frac{d-1}{2}\right)}{(d-2) ! \Gamma(1+d / 2)} .
$$

We know by Christiansen [5, Lemma 3.3] that

Lemma 2.6. The function $h_{d}(\theta)$ is of class $\mathscr{C}^{1}$ on $[0, \pi]$. Moreover, we have

$$
h_{d}^{\prime}(0+)=\lim _{\theta \rightarrow 0+} h_{d}^{\prime}(\theta)=e_{d} \quad \text { and } \quad h_{d}^{\prime}(\pi-0)=\lim _{\theta \rightarrow \pi-0} h_{d}^{\prime}(\theta)=-e_{d} .
$$

Here, $h_{d}^{\prime}(0+), h_{d}^{\prime}(\pi-0)$ denote respectively the right and left derivatives of $h_{d}$ at 0 and $\pi$. 
The measures involved in our main results are supported by the lower half-plane. However, it is more convenient to work with measures which are symmetric with respect to the real line because their potentials are easier to compute, see for instance Lemma 2.5. This is the reason why we introduce the following notions.

First, we extend the function $\kappa(z)$ defined in the Introduction on $\mathbb{C}_{-}$to $\mathbb{C} \backslash \mathbb{R}$ using the equation $\kappa(z)=\kappa(\bar{z})$. Define

$$
H_{Z}(z):= \begin{cases}0 & \text { for } z \in \mathbb{C}_{+} \\ c_{d}^{-1}|z|^{d} h_{d}(|\theta|) & \text { for } z \in \overline{\mathbb{C}}_{-}\end{cases}
$$

and

$$
H(z):=c_{d}^{-1}|z|^{d} h_{d}(|\theta|)=H_{Z}(z)+H_{Z}(\bar{z})= \begin{cases}H_{Z}(\bar{z}) & \text { for } z \in \overline{\mathbb{C}}_{+} \\ H_{Z}(z) & \text { for } \quad z \in \overline{\mathbb{C}}_{-},\end{cases}
$$

where $-\pi \leq \theta \leq \pi$ is the argument of $z$.

Lemma 2.7. We have

$$
d d^{c} H(z)=\frac{i}{2} \kappa(z) d z \wedge d \bar{z} \quad \text { on } \quad \mathbb{C} \backslash \mathbb{R} .
$$

Moreover, $\kappa(z)$ is an analytic real function which satisfies

$$
\kappa(t z)=t^{d-2} \kappa(z) \quad \text { and } \quad \kappa(z)=O\left(|y|^{1 / 2}|z|^{d-5 / 2}\right) \quad \text { as } z \rightarrow \infty .
$$

In particular, when $|z|$ is bounded, $\kappa(z)$ is bounded and $\kappa(z)=O\left(|y|^{1 / 2}\right)$ as $y$ tends to 0 .

Proof. Note that $\frac{i}{2} d z \wedge d \bar{z}=d x \wedge d y$ is the area form associated to the Lebesgue measure on $\mathbb{C}$. Observe that since $\kappa(z)$ is invariant by the maps $z \mapsto \pm \bar{z}$, it is enough to consider the case where $0<\theta \leq \pi / 2$. Recall that

$$
\Delta=\frac{\partial^{2}}{\partial r^{2}}+\frac{1}{r} \frac{\partial}{\partial r}+\frac{1}{r^{2}} \frac{\partial^{2}}{\partial \theta^{2}} \quad \text { and } \quad H(z)=c_{d}^{-1} r^{d} h_{d}(\theta) \quad \text { for } \quad z \in \mathbb{C}_{+} .
$$

Therefore, we have using the definition of $\kappa(z)$

$$
d d^{c} H(z)=\frac{i}{\pi} \partial \bar{\partial} H(z)=\frac{i}{4 \pi} \Delta H(z) d z \wedge d \bar{z}=\frac{i}{2} \kappa(z) d z \wedge d \bar{z} .
$$

This gives us the first identity.

The second identity is a direct consequence of the definition of $\kappa(z)$. We prove now the next estimate in the lemma using the previous ones and will see in the proof that $\kappa(z)$ is analytic real. Recall from [27] that the set $\Sigma:=\left\{z \in \mathbb{C}_{+}: \operatorname{Re} \rho(z)=0\right\}$ is a smooth analytic real curve intercepting the real line $\mathbb{R}$ at two points 1 and -1 . In polar coordinates $(r, \theta)$, it is given by an equation $r=r_{0}(\theta)$ with $0<\theta<\pi$, where $r_{0}(\theta)$ can be extended to an analytic real function in a neighbourhood of $[0, \pi]$. For $z=r e^{i \theta}$, we have $\operatorname{Re} \rho(z)<0$ is and only if $r>r_{0}(\theta)$. Moreover, we have $r_{0}(\theta)>1 / 2$ for $\theta \in[0, \pi]$. Define $s_{0}(z):=r_{0}(\theta)|z|^{-1}$. Using (2.2), (2.6) and the variable $s:=|z|^{-1} t$, we have

$$
H(z)=\frac{4}{c_{d}(d-2) !} \int_{0}^{\infty} \frac{\max (-\operatorname{Re} \rho(s z), 0)}{s^{d+1}} d s=-\frac{4}{c_{d}(d-2) !} \operatorname{Re} \int_{s_{0}(z)}^{\infty} \frac{\rho(s z)}{s^{d+1}} d s .
$$

Using the first identity and the fact that $\partial^{2} / \partial z \partial \bar{z}$ is a real operator, we have

$$
\kappa(z)=2 \frac{\partial^{2} H(z)}{\partial z \partial \bar{z}}=-\frac{8}{c_{d}(d-2) !} \operatorname{Re} \frac{\partial^{2}}{\partial z \partial \bar{z}} \int_{s_{0}(z)}^{\infty} \frac{\rho(s z)}{s^{d+1}} d s .
$$


It is clear now that $\kappa(z)$ is a real analytic function. We continue the proof of the last identity in the lemma. By the second identity in the lemma, it is enough to show that $\kappa(z)=O\left(y^{1 / 2}\right)$ when $|z|=1$ and $z \rightarrow 1$, or equivalently, the last second order derivative satisfies the same property.

Now, since $\rho(z)$ is holomorphic, its partial derivative in $\bar{z}$ vanishes. We deduce that

$$
\frac{\partial^{2}}{\partial z \partial \bar{z}} \int_{s_{0}(z)}^{\infty} \frac{\rho(s z)}{s^{d+1}} d s=-\frac{\partial}{\partial z}\left[\frac{\rho\left(s_{0}(z) z\right)}{s_{0}(z)^{d+1}} \frac{\partial s_{0}(z)}{\partial \bar{z}}\right]
$$

Recall that $s_{0}(z) z$ belongs to the curve $\Sigma$ and when $z \rightarrow 1$ we also have $s_{0}(z) z \rightarrow 1$. Since $r_{0}(\theta)$ is analytic in a neighbourhood of $[0, \pi]$, it is enough to check that $|\rho(z)|+\left|\rho^{\prime}(z)\right|=$ $O\left(|z-1|^{1 / 2}\right)$ when $z \rightarrow 1$. This property is clear because by (2.1), for $z \rightarrow 1$, we have

$$
\rho(z)=-\log z-\frac{1}{2}\left(1-z^{2}\right)+O\left(\left|1-z^{2}\right|^{3 / 2}\right) \quad \text { and } \quad \rho^{\prime}(z)=O\left(\left|1-z^{2}\right|^{1 / 2}\right) .
$$

The lemma follows.

Lemma 2.8. The functions $H_{Z}, H$ are subharmonic on $\mathbb{C}$ and $\mu^{-}, \mu_{\mathrm{MZ}}, d d^{c} H$ are positive measures on $\mathbb{C}$. Define the positive measure $\mu$ on $\mathbb{C}$ by $\mu:=d d^{c} H$. Let $\mu^{+}$be the image of $\mu^{-}$by the map $z \mapsto \bar{z}$. Then we have

$$
d d^{c} H_{Z}=\mu^{-}+\mu^{0}=\mu_{\mathrm{MZ}} \quad \text { and } \quad \mu=\mu^{+}+\mu^{-}+2 \mu^{0} .
$$

Proof. For the first assertion, it is enough to show that $H_{Z}(z)$ is subharmonic because this property implies that $H_{Z}(\bar{z})$ and hence $H(z)$ are also subharmonic. Using properties of $h_{d}$ and Lemma 2.6, we see that $H_{Z}(z)$ is continuous, non-negative on $\overline{\mathbb{C}}_{+}$and vanishes on $\mathbb{R}$. By Lemma 2.4, it is enough to check that $H_{Z}(z)$ is subharmonic on $\mathbb{C}_{+}$.

Using (2.2), (2.6) and the variable $s:=|z|^{-1} t$, we have for $z \in \mathbb{C}_{+}$

$$
H_{Z}(z)=\frac{4}{c_{d}(d-2) !} \int_{0}^{\infty} \frac{\max (-\operatorname{Re} \rho(s z), 0)}{s^{d+1}} d s .
$$

Since the function $z \mapsto \rho(s z)$ is holomorphic, the function $z \mapsto-\operatorname{Re} \rho(s z)$ is harmonic and the function $z \mapsto \max (-\operatorname{Re} \rho(s z), 0)$ is subharmonic. We easily deduce that $H_{Z}(z)$ is subharmonic and hence $H$ is also subharmonic.

We prove now the two identities in the lemma. Since $H_{Z}$ vanishes on $\mathbb{C}_{+}$, the measure $\mu_{\mathrm{MZ}}$ also vanishes there. It follows from Lemma 2.7 that $d d^{c} H_{Z}=\mu^{-}$on $\mathbb{C}_{-}$. If $m$ denotes the restriction of $d d^{c} H_{Z}$ to $\mathbb{R}$, then we have $d d^{c} H_{Z}=\mu^{-}+m$. We also deduce from (2.7) that $\mu=\mu^{+}+\mu^{-}+2 m$. So $2 m$ is the restriction of $\mu=d d^{c} H$ to $\mathbb{R}$ and it remains to check that $m=\mu^{0}$.

By Lemma 2.6 and (2.7), the function $H$ is $\mathscr{C}^{1}$ on $\overline{\mathbb{C}}_{+}$. Moreover, on $\overline{\mathbb{C}}_{+}$, we have for $x>0$

$$
\frac{\partial H}{\partial y}(x)=c_{d}^{-1} x^{d} x^{-1} h_{d}^{\prime}(0)=c_{d}^{-1} x^{d-1} e_{d}
$$

and for $x<0$

$$
\frac{\partial H}{\partial y}(x)=c_{d}^{-1}|x|^{d} x^{-1} h_{d}^{\prime}(\pi-0)=c_{d}^{-1}|x|^{d-1} e_{d} .
$$

It is also easy to check that this derivative vanishes at 0 . Now, the result follows from the second assertion of Lemma 2.5 and the definition of $\mu^{0}$ in the Introduction. 
Lemma 2.9. For $0 \leq \theta_{1}<\theta_{2} \leq \pi$, let $\Omega\left(\theta_{1}, \theta_{2}\right)$ be as in Corollary 1.2. Then we have

$$
\mu_{\mathrm{MZ}}\left(\Omega\left(\theta_{1}, \theta_{2}\right)\right)=\frac{1}{2 \pi d c_{d}}\left[h_{d}^{\prime}\left(\theta_{2}\right)-h_{d}^{\prime}\left(\theta_{1}\right)+d^{2} \int_{\theta_{1}}^{\theta_{2}} h_{d}(\theta) d \theta\right]
$$

where we replace $h_{d}^{\prime}\left(\theta_{1}\right)$ and $h_{d}^{\prime}\left(\theta_{2}\right)$ by $h_{d}^{\prime}(0+)$ and $h_{d}^{\prime}(\pi-0)$ respectively when $\theta_{1}=0$ and $\theta_{2}=\pi$. Moreover, we have

$$
\mu_{\mathrm{MZ}}([0,1])=\mu_{\mathrm{MZ}}([-1,0])=\frac{e_{d}}{2 \pi d c_{d}} \quad \text { and } \quad \mu_{\mathrm{MZ}}(\mathbb{D})=\mu_{\mathrm{MZ}}\left(\overline{\mathbb{D}}_{-}\right)=1 .
$$

Proof. We have

$$
\begin{aligned}
\mu_{\mathrm{MZ}}\left(\Omega\left(\theta_{1}, \theta_{2}\right)\right) & =\int_{0<r<1, \theta_{1}<\theta<\theta_{2}} \kappa(z) d x d y \\
& =\int_{0<r<1, \theta_{1}<\theta<\theta_{2}} \frac{1}{2 \pi c_{d}} r^{d-2}\left[d^{2} h_{d}(\theta)+h_{d}^{\prime \prime}(\theta)\right] r d r d \theta .
\end{aligned}
$$

The first identity in the lemma follows easily.

For the second assertion in the lemma, we have

$$
\mu_{\mathrm{MZ}}([0,1])=\mu^{0}([0,1])=\int_{0}^{1} \frac{e_{d}}{2 \pi c_{d}} x^{d-1} d x=\frac{e_{d}}{2 \pi d c_{d}} .
$$

Similar identities also hold for $[-1,0]$. Recall that $\mu_{\mathrm{MZ}}$ has no mass on $\mathbb{C}_{+}$and is absolutely continuous with respect to the Lebesgue measure on $\mathbb{C}_{-}$. Therefore, we have

$$
\mu_{\mathrm{MZ}}(\mathbb{D})=\mu_{\mathrm{MZ}}\left(\overline{\mathbb{D}}_{-}\right)=\mu^{-}\left(\mathbb{D}_{-}\right)+\mu^{0}([-1,1])=\mu^{-}\left(\mathbb{D}_{-}\right)+\frac{e_{d}}{\pi d c_{d}} .
$$

Using the first identity in the lemma, (2.3) and Lemma 2.6, we get

$$
\mu_{\mathrm{MZ}}\left(\mathbb{D}_{-}\right)=\mu^{-}\left(\mathbb{D}_{-}\right)=\frac{1}{2 \pi d c_{d}}\left[-2 e_{d}+2 \pi d c_{d}\right]=1-\frac{e_{d}}{\pi d c_{d}} .
$$

It follows that $\mu_{\mathrm{MZ}}(\mathbb{D})=\mu_{\mathrm{MZ}}\left(\overline{\mathbb{D}}_{-}\right)=1$. This completes the proof of the lemma.

\section{Distribution OF THE RESONANCES}

In this section, we will prove the main results stated in the Introduction. Recall that in order to simplify the proof we will symmetrize the measures and potentials with respect to the real axis. Let $S_{V}(z)$ be the scattering matrix associated to $R_{V}(z)$ and $s_{V}(z):=$ $\operatorname{det} S_{V}(z)$, see e.g. [10]. Recall that $s_{V}(z) s_{V}(-z)=1$ and if $s_{V}$ has poles in the closed upper half-plane $\overline{\mathbb{C}}_{+}$, it has only finitely many. Moreover, with at most finitely many exceptions, the zeros of $s_{V}(z)$ coincide with the poles of $R_{V}(-z)$, and the multiplicities agree. Therefore, our study uses in a crucial way the function $s_{V}(z)$. The following result was obtained in [9], see also [27, Theorem 5].

Proposition 3.1. There is a constant $A>0$ depending only on $d, a$ and $V$ such that

$$
\log \left|s_{V}\left(r e^{i \theta}\right)\right| \leq h_{d}(\theta) a^{d} r^{d}+A r^{d-1} \log r
$$

for all $r \geq A$ and $\theta \in[0, \pi]$.

We also have the following estimate. 
Proposition 3.2. We have for $r \geq 0$

$$
\left|N_{V}(r)-\frac{1}{2 \pi} \int_{0}^{\pi} \log \right| s_{V}\left(r e^{i \theta}\right)|d \theta| \leq A r^{d-1}+A
$$

where $A>0$ is a constant depending only on $d, a$ and $V$.

Proof. By Christiansen [5, (3.2)] and Stefanov [27, Lemma 2], the estimate holds for $r$ large enough, see also Froese [12], Petkov-Zworski [17]. Since $s_{V}(z) s_{V}(-z)=1$, we have $s_{V}(0) \neq 0$ and therefore, the estimate holds for $r$ small enough. Finally, when $r$ is bounded by two positive constants, since $s_{V}$ is a meromorphic function, the integral in the lemma is bounded. We easily deduce the proposition by taking $A$ large enough.

The following estimate due to Christiansen [5, Lemma 3.1] will be needed.

Lemma 3.3. There is a constant $A$ depending only on $d$, $a$ and $V$ such that

$$
\left|\frac{d}{d z} \log s_{V}(z)\right| \leq A|z|^{d-2}
$$

for $z \in \mathbb{R}$ with $|z|$ large enough.

Finally, we will also use the following result which relates the asymptotic behavior of $n_{V}(r)$ and $N_{V}(r)$. It was obtained in [27, Lemma 1] and [9, Prop. 5.1].

Lemma 3.4. Let $\nu$ and $A$ be two constants such that $0<\nu<d$ and $A>0$. Then the following holds.

(1) $n_{V}(r)=A r^{d}+o\left(r^{d}\right)$ as $r \rightarrow \infty$ if and only if $N_{V}(r)=\frac{A r^{d}}{d}+o\left(r^{d}\right)$ as $r \rightarrow \infty$.

(2) If $n_{V}(r)=A r^{d}+O\left(r^{d-\nu}\right)$ as $r \rightarrow \infty$, then $N_{V}(r)=\frac{A r^{d}}{d}+O\left(r^{d-\nu}\right)$ as $r \rightarrow \infty$.

(3) If $N_{V}(r)=A r^{d}+O\left(r^{d-\nu}\right)$ as $r \rightarrow \infty$, then $n_{V}(r)=\frac{A r^{d}}{d}+O\left(r^{d-\nu / 2}\right)$ as $r \rightarrow \infty$.

For $r>0$, consider the following function

$$
u_{V, r}(z):=\left\{\begin{array}{lll}
\frac{1}{c_{d} a^{d} r^{d}} \log \left|s_{V}(-r \bar{z})\right| & \text { for } & z \in \overline{\mathbb{C}}_{+}, \\
\frac{1}{c_{d} a^{d} r^{d}} \log \left|s_{V}(-r z)\right| & \text { for } & z \in \overline{\mathbb{C}}_{-} .
\end{array}\right.
$$

Proposition 3.5. The following properties hold.

(1) If $V$ belongs to the Christiansen class $\mathfrak{M}_{a}$, then

$$
\sup _{s \geq 1} s^{-d-2}\left\|u_{V, r}-H\right\|_{L^{1}(\mathbb{D}(s))} \rightarrow 0 \quad \text { as } \quad r \rightarrow \infty ;
$$

(2) If $V$ belongs to the class $\mathfrak{M}_{a}^{\nu}$ for some $0<\nu \leq 1$, then for every $\eta>0$

$$
\sup _{s \geq 1} s^{-d-2}\left\|u_{V, r}-H\right\|_{L^{1}(\mathbb{D}(s))}=O\left(r^{-\nu+\eta}\right) \quad \text { as } \quad r \rightarrow \infty .
$$

Proof. (1) It is enough to consider $r$ large enough. Define

$$
\eta(r):=\frac{1}{c_{d} a^{d} r^{d}}\left|N_{V}(r)-\frac{c_{d} a^{d} r^{d}}{d}\right| .
$$

Since $V$ is a function in the Christiansen class $\mathfrak{M}_{a}$, by the first assertion of Lemma 3.4, we have that $\eta(r)$ tends to 0 when $r$ tends to infinity. By Proposition 3.2, we have

$$
\left|\frac{1}{2 \pi} \int_{0}^{\pi} \log \right| s_{V}\left(r e^{i \theta}\right)\left|d \theta-\frac{c_{d} a^{d} r^{d}}{d}\right| \leq c_{d} a^{d} r^{d} \eta(r)+A r^{d-1}+A
$$


for $r \geq 0$. Using (3.1), we obtain that

$$
\left|\frac{1}{2 \pi} \int_{0}^{\pi} u_{V, r}\left(t e^{i \theta}\right) d \theta-\frac{t^{d}}{d}\right| \leq t^{d} \eta(t r)+A^{\prime} t^{d-1} r^{-1}+A^{\prime} r^{-d},
$$

for some constant $A^{\prime}>0$. Therefore, by (2.3), we can rewrite the last line as

$$
\left|\frac{1}{2 \pi} \int_{0}^{\pi}\left[u_{V, r}\left(t e^{i \theta}\right)-\frac{h_{d}(\theta) t^{d}}{c_{d}}\right] d \theta\right| \leq t^{d} \eta(t r)+A^{\prime} t^{d-1} r^{-1}+A^{\prime} r^{-d}
$$

or equivalently, by (2.7),

$$
\left|\frac{1}{2 \pi} \int_{0}^{\pi}\left[u_{V, r}\left(t e^{i \theta}\right)-H\left(t e^{i \theta}\right)\right] d \theta\right| \leq t^{d} \eta(t r)+A^{\prime} t^{d-1} r^{-1}+A^{\prime} r^{-d} .
$$

Finally, using the polar coordinates and Fubini's theorem, we deduce that

$$
\left\|u_{V, r}-H\right\|_{L^{1}(\mathbb{D}(s))} \leq \int_{0}^{s}\left[t^{d} \eta(t r)+A^{\prime} t^{d-1} r^{-1}+A^{\prime} r^{-d}\right] t d t \leq \int_{0}^{s} t^{d+1} \eta(t r) d t+A^{\prime \prime} s^{d+1} r^{-1}
$$

for some constant $A^{\prime \prime}>0$. By a simple change of variable, we see that the last integral is equal to $s^{d+2} \gamma(r s)$ with

$$
\gamma(r s):=(r s)^{-d-2} \int_{0}^{r s} t^{d+1} \eta(t) d t
$$

Since $\eta(t)$ tends to 0 as $t$ tends to infinity, $\gamma(r s)$ converges to 0 as $r s$ tends to infinity. In particular, $\gamma(r s)$ tends to 0 when $r$ tends to infinity, uniformly in $s$ because $s \geq 1$. This completes the proof of the first assertion of the proposition.

(2) Assume now that $V$ is in $\mathfrak{M}_{a}^{\nu}$. We can also assume that $\eta<\nu$. By the second assertion of Lemma 3.4, we have $\eta(r s) \leq A_{\eta}(r s)^{-\nu+\eta}$ for some constant $A_{\eta}>0$. It follows that

$$
\gamma(r s) \leq(r s)^{-d-2} A_{\eta}(r s)^{d+2-\nu+\eta} \leq A_{\eta} r^{-\nu+\eta}
$$

since $s \geq 1$. The proposition follows.

Recall that $s_{V}(z) s_{V}(-z)=1$ for $z \in \mathbb{C}$ and $s_{V}$ has only a finite number of poles in the closed upper half-plane $\overline{\mathbb{C}}_{+}$. We denote them by $z_{1}, \ldots, z_{m}$, where each pole is repeated according to its multiplicity. It is convenient to modify the functions $s_{V}$ and $u_{V, r}$ slightly. Define

$$
\hat{s}_{V}(z):=\prod_{j=1}^{m} \frac{z-z_{j}}{z+z_{j}} s_{V}(z) .
$$

We see that $\hat{s}_{V}$ is a holomorphic function on an open neighborhood of $\overline{\mathbb{C}}_{+}$with neither zeros nor poles on the real line $\mathbb{R}$. Define also

$$
\hat{u}_{V, r}(z):=\left\{\begin{array}{lll}
\frac{1}{c_{d} a^{d} r^{d}} \log \left|\hat{s}_{V}(-r \bar{z})\right| & \text { for } & z \in \overline{\mathbb{C}}_{+} \\
\frac{1}{c_{d} a^{d} r^{d}} \log \left|\hat{s}_{V}(-r z)\right| & \text { for } & z \in \overline{\mathbb{C}}_{-} .
\end{array}\right.
$$

Lemma 3.6. There is a constant $A>0$ such that

$$
\left|\frac{d}{d z} \log \hat{s}_{V}(z)\right| \leq A\left(|z|^{d-2}+1\right) \quad \text { for } \quad z \in \mathbb{R} .
$$


Proof. By Lemma 3.3 and (3.2), there are constants $A>0$ and $R>0$ such that the above inequality holds for $z \in \mathbb{R}$ such that $|z| \geq R$. On the other hand, $\hat{s}_{V}$ is holomorphic and does not vanish in a neighbourhood of $\mathbb{R}$. Therefore, the same inequality holds for $z \in[-R, R]$ provided that $A$ is large enough. The lemma follows.

Lemma 3.7. We have the following properties.

(1) If $V$ belongs to the Christiansen class $\mathfrak{M}_{a}$, then

$$
\sup _{s \geq 1} s^{-d-2}\left\|\hat{u}_{V, r}-H\right\|_{L^{1}(\mathbb{D}(s))} \rightarrow 0 \quad \text { as } \quad r \rightarrow \infty ;
$$

(2) If $V$ belongs to the class $\mathfrak{M}_{a}^{\nu}$ for some $0<\nu \leq 1$, then for every $\eta>0$,

$$
\sup _{s \geq 1} s^{-d-2}\left\|\hat{u}_{V, r}-H\right\|_{L^{1}(\mathbb{D}(s))}=O\left(r^{-\nu+\eta}\right) \quad \text { as } \quad r \rightarrow \infty .
$$

Proof. By (3.1) and (3.3), we have

$$
\begin{aligned}
\hat{u}_{V, r}(z)-u_{V, r}(z) & =\frac{1}{c_{d} a^{d} r^{d}} \sum_{j=1}^{m} \log \left|r z-z_{j}\right|-\log \left|r z+z_{j}\right| \\
& =\frac{1}{c_{d} a^{d} r^{d}} \sum_{j=1}^{m} \log \left|z-r^{-1} z_{j}\right|-\log \left|z+r^{-1} z_{j}\right| .
\end{aligned}
$$

So for $r$ large enough, we have $\left|r^{-1} z_{j}\right| \leq 1$ and hence the $L^{1}$-norm of $\log \left|z \pm r^{-1} z_{j}\right|$ on $\mathbb{D}(s)$ is bounded by the $L^{1}$-norm of $\log |z|$ on $\mathbb{D}(s+1)$. The last one is bounded by a constant times $s^{2} \log (s+1)$. Therefore, the lemma follows from the last identities and Proposition 3.5.

Now, define $\hat{\mu}_{V, r}:=d d^{c} \hat{u}_{V, r}$. Since $\hat{s}_{V}$ has no pole on $\overline{\mathbb{C}}_{+}$and no zero on $\mathbb{R}$, the function $\hat{u}_{V, r}$ is subharmonic on $\mathbb{C}_{+} \cup \mathbb{C}_{-}$and is symmetric with respect to $\mathbb{R}$. We can apply Lemma 2.5 to this function on a suitable neighbourhood $\Omega$ of $\mathbb{R}$ and deduce that $\hat{\mu}_{V, r}$ is a measure on $\mathbb{C}$. Denote also by $\hat{\mu}_{V, r}^{+}, \hat{\mu}_{V, r}^{-}$and $2 \hat{\mu}_{V, r}^{0}$ the restrictions of $\hat{\mu}_{V, r}$ to $\mathbb{C}_{+}, \mathbb{C}_{-}$and $\mathbb{R}$, respectively. Let $\mathfrak{Z}_{V}$ denote the set of zeros of $s_{V}(z)$ on $\mathbb{C}_{+}$where each point is repeated according to its multiplicity. This is also the zero set of $\hat{s}_{V}(z)$ on $\mathbb{C}_{+}$. We see that

$$
\hat{\mu}_{V, r}^{+}=\frac{1}{c_{d} a^{d} r^{d}} \sum_{z \in \mathfrak{Z}_{V}} \delta_{z / r} .
$$

Moreover, by definition of $\hat{u}_{V, r}, \hat{\mu}_{V, r}^{-}$is the image of $\hat{\mu}_{V, r}^{+}$by the map $z \mapsto \bar{z}$. So both $\hat{\mu}_{V, r}^{+}$ and $\hat{\mu}_{V, r}^{-}$are positive measures.

Lemma 3.8. Let $0<\gamma \leq 2$ be a constant and let $\Omega, \Omega^{\prime}$ be two open discs in $\mathbb{C}$ centered at 0 with $\Omega \Subset \Omega^{\prime}$. Then there is a constant $A>0$ independent of $r$ such that $A r^{-1} \operatorname{Leb}_{\mathbb{R}} \pm \hat{\mu}_{V, r}^{0}$ are positive measures on $\Omega$ and

$$
\operatorname{dist}_{\Omega, \gamma}\left(\hat{\mu}_{V, r}^{+}+\hat{\mu}_{V, r}^{-}, \mu\right) \leq A r^{-1}+A\left\|\hat{u}_{V, r}-H\right\|_{L^{1}\left(\Omega^{\prime}\right)}^{\gamma / 2} .
$$

In particular, the mass of $\hat{\mu}_{V, r}^{0}$ on $\Omega$ tends to 0 as $r$ tends to infinity.

Proof. It is clear that the last assertion is a consequence of the first one. Write $\Omega=\mathbb{D}(s)$ and $\Omega^{\prime}=\mathbb{D}\left(s^{\prime}\right)$ with $s<s^{\prime}$. Since $\hat{u}_{V, r}$ is symmetric with respect to $\mathbb{R}$, we can apply Lemma 2.5 to this function. Denote by $u$ the restriction of $\hat{u}_{V, r}$ to $\mathbb{D}\left(s^{\prime}\right) \cap \overline{\mathbb{C}}_{+}$as in Lemma 2.5, By Lemma 3.6, we have $\left|\frac{\partial u}{\partial y}\right| \leq A r^{-1}$ on $\mathbb{D}\left(s^{\prime}\right) \cap \mathbb{R}$ for some constant $A>0$ 
independent of $r$. Then, using Lemma 2.5, we see that $A r^{-1} \operatorname{Leb}_{\mathbb{R}} \pm \hat{\mu}_{V, r}^{0}$ are positive measures on $\mathbb{D}\left(s^{\prime}\right)$. The first assertion of the lemma follows.

For the estimate in the lemma, by (1.5), we only need to check that

$$
\operatorname{dist}_{\mathbb{D}\left(s^{\prime}\right), 2}\left(\hat{\mu}_{V, r}^{+}+\hat{\mu}_{V, r}^{-}, \mu\right) \leq A r^{-1}+A\left\|\hat{u}_{V, r}-H\right\|_{L^{1}\left(\mathbb{D}\left(s^{\prime}\right)\right)}
$$

or equivalently if $\varphi$ is a $\mathscr{C}^{2}$ function with compact support in $\mathbb{D}\left(s^{\prime}\right)$ with $\|\varphi\|_{\mathscr{C}^{2}} \leq 1$, we need to show that

$$
\left|\left\langle\hat{\mu}_{V, r}^{+}+\hat{\mu}_{V, r}^{-}-\mu, \varphi\right\rangle\right| \leq A r^{-1}+A\left\|\hat{u}_{V, r}-H\right\|_{L^{1}\left(\mathbb{D}\left(s^{\prime}\right)\right)} .
$$

Consider the function $u^{*}(z):=\hat{u}_{V, r}(z)+2 \pi A r^{-1}|y|$. It follows from the above arguments that $\mu^{*}:=d d^{c} u^{*}-\left(\hat{\mu}_{V, r}^{+}+\hat{\mu}_{V, r}^{-}\right)$is a positive measure on $\mathbb{D}\left(s^{\prime}\right)$ of mass bounded by a constant times $r^{-1}$. Therefore, since $\mu=d d^{c} H$ and $d d^{c} \varphi$ is bounded, we have

$$
\begin{aligned}
\left|\left\langle\hat{\mu}_{V, r}^{+}+\hat{\mu}_{V, r}^{-}-\mu, \varphi\right\rangle\right| & \leq\left|\left\langle\mu^{*}, \varphi\right\rangle\right|+\left|\left\langle d d^{c} u^{*}-d d^{c} H, \varphi\right\rangle\right| \\
& \lesssim r^{-1}+\left|\left\langle u^{*}-H, d d^{c} \varphi\right\rangle\right| \\
& \lesssim r^{-1}+\left\|u^{*}-H\right\|_{L^{1}\left(\mathbb{D}\left(s^{\prime}\right)\right)} \\
& \lesssim r^{-1}+\left\|\hat{u}_{V, r}-H\right\|_{L^{1}\left(\mathbb{D}\left(s^{\prime}\right)\right) .}
\end{aligned}
$$

So the estimate in the lemma holds for some constant $A>0$ large enough. This completes the proof of the lemma.

End of the proof of Theorem 1.1. By Lemmas 3.7 and 3.8, $\operatorname{dist}_{\Omega, 2}\left(\hat{\mu}_{V, r}^{+}+\hat{\mu}_{V, r}^{-}, \mu\right)$ tends to 0 as $r$ tends to infinity for any disc $\Omega$ of center 0 . It follows that $\hat{\mu}_{V, r}^{+}+\hat{\mu}_{V, r}^{-}$tends to $\mu$ weakly. Recall that $\hat{\mu}_{V, r}^{+}$and $\hat{\mu}_{V, r}^{-}$have supports in $\mathbb{C}_{+}$and $\mathbb{C}_{-}$respectively. Moreover, they are symmetric each to other with respect to $\mathbb{R}$. Recall also from Lemma 2.8 that $\mu=\mu^{+}+\mu^{-}+2 \mu^{0}$ where $\mu^{+}, \mu^{-}, 2 \mu^{0}$ are the restrictions of $\mu$ to $\mathbb{C}_{+}, \mathbb{C}_{-}$and $\mathbb{R}$ respectively. Therefore, the fact that $\hat{\mu}_{V, r}^{+}+\hat{\mu}_{V, r}^{-}$tends to $\mu$ implies that $\hat{\mu}_{V, r}^{-}$converges weakly to $\mu^{-}+\mu^{0}$. The last measure is equal to $\mu_{\mathrm{MZ}}$.

Recall that with at most finitely many of exceptions, a point $z$ is a resonance, i.e. a pole of $R_{V}$, if and only if $-z$ is a zero of $\hat{s}_{V}$ and their multiplicities agree. Therefore, the measure $\mu_{V, r}^{\Re}-\hat{\mu}_{V, r}^{-}$is a finite combination of atoms with total mass bounded by a constant times $r^{-d}$. We deduce that $\mu_{V, r}^{\Re}$ and $\hat{\mu}_{V, r}^{-}$have the same limit $\mu_{\mathrm{MZ}}$ when $r$ tends to infinity. This is the first assertion in the theorem.

Now, consider the second assertion in the theorem. Since there are finitely many of resonances in $\overline{\mathbb{C}}_{+}$, it is enough to prove the statement for $W=\Omega$ and for $W=\bar{\Omega}$. Since $n_{V, W}(r)=c_{d} a^{d} r^{d} \mu_{V, r}^{\Re}(W)$, we easily deduce the second assertion in the theorem from the first one by using Lemma 2.1.

End of the proof of Corollary 1.2. Consider first the case where $0<\theta_{1}<\theta_{2}<\pi$. By Theorem 1.1 applied to $\Omega:=\Omega\left(\theta_{1}, \theta_{2}\right)$, we have

$$
n_{V, \Omega}(r)=c_{d} a^{d} \mu_{\mathrm{MZ}}(\Omega) r^{d}+o\left(r^{d}\right) .
$$

We easily deduce the result from Lemma 2.9.

Consider now the case where $\theta_{1}=0$ and $\theta_{2}<\pi$. The other cases can be treated in the same way. Let $\Omega^{*}$ be the open sector of the unit disc which is the union of $\Omega$, its symmetry 
with respect to $\mathbb{R}$ and the radius $(-1,0)$. Applying Theorem 1.1 to $\Omega^{*}$ instead of $\Omega$ and using that $\mu_{\mathrm{MZ}}\left(\mathbb{C}_{+}\right)=0$, we obtain

$$
n_{V, \Omega^{*}}(r)=c_{d} a^{d}\left[\mu_{\mathrm{MZ}}((-1,0))+\mu_{\mathrm{MZ}}(\Omega)\right] r^{d}+o\left(r^{d}\right) .
$$

Since there are only finitely many of resonances in $\overline{\mathbb{C}}_{+}$, the last identity still holds if we replace $\Omega^{*}$ by $\Omega$. Lemmas 2.6 and 2.9 imply the result.

End of the proof of Theorem 1.3. We have seen in the proof of Theorem 1.1 that the mass of $\mu_{V, r}^{\Re}-\hat{\mu}_{V, r}^{-}$is bounded by a constant times $r^{-d}$. Therefore, for simplicity, we will prove the first assertion in the theorem for $\hat{\mu}_{V, r}^{-}$instead of $\mu_{V, r}^{\Re}$. By (1.5), we can assume that $\gamma=1$. Let $\varphi$ be a function with compact support in $\Omega$ such that $\|\varphi\|_{\mathscr{C}^{1}} \leq 1$. We need to show that $\left|\left\langle\hat{\mu}_{V, r}^{-}-\mu_{\mathrm{MZ}}, \varphi\right\rangle\right| \lesssim r^{-\nu / 2+\eta}$ for any constant $\eta>0$.

Observe that $\Omega \cap \mathbb{R}$ is either empty or a finite union of open bounded intervals. Define $\hat{\Omega}_{-}:=\Omega \cap \mathbb{C}_{-}$. We add here a hat in order to avoid any confusion. Let $\hat{\Omega}_{+}$be the symmetry of $\hat{\Omega}_{-}$with respect to $\mathbb{R}$ and let $\hat{\Omega}$ be the union of $\hat{\Omega}_{+}, \hat{\Omega}_{-}$and $\Omega \cap \mathbb{R}$. So $\hat{\Omega}$ is a bounded open set, symmetric with respect to $\mathbb{R}$, whose boundary is piecewise smooth and transverse to $\mathbb{R}$. Define $\hat{\varphi}(z):=\varphi(z)$ for $z$ in $\hat{\Omega}_{-}$or in $\Omega \cap \mathbb{R}$ and $\hat{\varphi}(z):=\varphi(\bar{z})$ if $z \in \hat{\Omega}_{+}$. Using the description of $\mu$ in Lemma 2.8 , we see that the desired estimate is equivalent to the inequality $\left|\left\langle\hat{\mu}_{V, r}^{+}+\hat{\mu}_{V, r}^{-}-\mu, \hat{\varphi}\right\rangle\right| \lesssim r^{-\nu / 2+\eta}$.

We apply the second assertion of Lemma 3.7 and Lemma 3.8 for $\gamma=1$ and for large enough discs. If $\psi$ is a function with compact support in $\hat{\Omega}$ such that $\|\psi\|_{\mathscr{C}^{1}} \leq 1$, then we have

$$
\left|\left\langle\hat{\mu}_{V, r}^{+}+\hat{\mu}_{V, r}^{-}-\mu, \psi\right\rangle\right| \lesssim r^{-1}+\left\|\hat{u}_{V, r}-H\right\|_{L^{1}(U)}^{1 / 2} \lesssim r^{-\nu / 2+\eta}
$$

for some disc $U$ large enough. The estimate still holds when $\psi$ is a Lipschitz function with Lipschitz constant smaller or equal to $1,|\psi| \leq 1$, because using the standard convolution we can approximate it uniformly by smooth functions with $\mathscr{C}^{1}$-norm at most equal to 1 . So we can apply the identity for the above function $\hat{\varphi}$ instead of $\psi$ and this completes the proof of the first assertion.

Observe that we can write $\Omega$ as the disjoint union of a finite number of subsets so that each of them is either a nice open set as in Lemma 2.3 or the union of such a set with some smooth pieces of boundary. We can also choose these sets so that these pieces of boundaries are transverse to the real line. Thus, for simplicity, we can assume that $\Omega$ is the nice open set described in Lemma 2.3, Let $\hat{\Omega}$ be defined as above.

We apply Lemma 2.2 to $\hat{\Omega}$ instead of $\Omega$, and for $u_{2}:=\hat{u}_{V, r}, u_{1}:=H, \epsilon:=\left\|\hat{u}_{V, r}-H\right\|_{L^{1}(U)}^{1 / 3}$. Recall that $d d^{c} \hat{u}_{V, r}=\hat{\mu}_{V, r}^{+}+\hat{\mu}_{V, r}^{-}+2 \hat{\mu}_{V, r}^{0}$ and $d d^{c} H=\mu=\mu^{+}+\mu^{-}+2 \mu^{0}$. Moreover, by Lemma 3.7, we have $\left\|\hat{u}_{V, r}-H\right\|_{L^{1}(U)} \lesssim r^{-\nu+\eta}$. Observe also that since the boundary of $\hat{\Omega}$ is piecewise smooth and transverse to $\mathbb{R}$, we have $\mu\left((b \hat{\Omega})_{\epsilon}\right)=O(\epsilon)$. Therefore, applying Lemma 2.2 gives

$$
\left|\hat{\mu}_{V, r}^{+}(\hat{\Omega})+\hat{\mu}_{V, r}^{-}(\hat{\Omega})+2 \hat{\mu}_{V, r}^{0}(\hat{\Omega})-\mu^{+}(\hat{\Omega})-\mu^{-}(\hat{\Omega})-2 \mu^{0}(\hat{\Omega})\right| \lesssim r^{-\nu / 3+\eta}
$$

for every $\eta>0$, or equivalently,

$$
\left|2 \hat{\mu}_{V, r}^{-}(\Omega)+2 \hat{\mu}_{V, r}^{0}(\Omega)-2 \mu_{\mathrm{MZ}}(\Omega)\right| \lesssim r^{-\nu / 3+\eta} .
$$

This, together with the mass estimate $\hat{\mu}_{V, r}^{0}(\Omega) \leq A r^{-1} \operatorname{Leb}_{\mathbb{R}}(\Omega)$ obtained in Lemma 3.8, imply

$$
\left|\hat{\mu}_{V, r}^{-}(\Omega)-\mu_{\mathrm{MZ}}(\Omega)\right| \lesssim r^{-\nu / 3+\eta} \quad \text { and hence } \quad\left|\mu_{V, r}^{\Re}(\Omega)-\mu_{\mathrm{MZ}}(\Omega)\right| \lesssim r^{-\nu / 3+\eta} \text {. }
$$


Using the last assertion in Lemma 2.2 , we obtain the same estimate for $\bar{\Omega}$ instead of $\Omega$.

Finally, since $n_{V, W}(r)=c_{d} a^{d} r^{d} \mu_{V, r}^{\Re}(W)$, the inequalities obtained above imply the last estimate in Theorem 1.3 for $W:=\Omega$ or $\bar{\Omega}$. Recall that there are only finitely many resonances in $\overline{\mathbb{C}}_{+}$. So the estimate holds for all $W$ such that $\Omega \cap \mathbb{C}_{-} \subset W \subset \bar{\Omega}$. This completes the proof of the theorem.

\section{REFERENCES}

[1] Christiansen T., Some lower bounds on the number of resonances in Euclidean scattering, Math. Res. Lett. 6 (1999), no. 2, 203-211.

[2] Christiansen T., Several complex variables and the distribution of resonances in potential scattering, Comm. Math. Phys. 259 (2005), no. 3, 711-728.

[3] Christiansen T., Schrödinger operators with complex-valued potentials and no resonances, Duke Math. J. 133 (2006), no. 2, 313-323.

[4] Christiansen T., Several complex variables and the order of growth of the resonance counting function in Euclidean scattering, Int. Math. Res. Not. 12 (2006), 36pp.

[5] Christiansen T., Schrödinger operators and the distribution of resonances in sectors, Anal. PDE $\mathbf{5}$ (2012), 961-982.

[6] Christiansen T., Hislop P. D., The resonance counting function for Schrödinger operators with generic potentials, Math. Res. Lett. 12 (2005), no. 5-6, 821-826.

[7] Dinh T.-C., Nguyên V.-A., Sibony N., Exponential estimates for plurisubharmonic functions and stochastic dynamics, J. Diff. Geom. 84 (2010), no. 3, 465-488.

[8] Dinh T.-C., Sibony N., Dynamics in several complex variables: endomorphisms of projective spaces and polynomial-like mappings. Holomorphic dynamical systems, 165-294, Lecture Notes in Math., 1998. Springer, Berlin, 2010.

[9] Dinh T.-C., Vu D.-V., Asymptotic number of scattering resonances for generic Schrödinger operators, Comm. Math. Phys. 326 (2014), no. 1, 185-208.

[10] Dyatlov S., Zworski M., Mathematical theory of scattering resonances, version 0.03, http://math. berkeley.edu/ zworski, book in progress.

[11] Froese R., Asymptotic distribution of resonances in one dimension, J. Differential Equations 137 (1997), no. 2, 251-272.

[12] Froese R., Upper bounds for the resonance counting function of Schrödinger operators in odd dimensions, Canad. J. Math. 50 (1998), no. 3, 538-546.

[13] Lelong P., Gruman L., Entire functions of several complex variables, 282, Springer-Verlag, Berlin, 1986.

[14] Melrose R.B., Scattering theory and the trace of the wave group, J. Funct. Anal. 45 (1982), 29-40.

[15] Melrose R.B., Polynomial bounds on the number of scattering poles, J. Funct. Anal. 53 (1983), 287303.

[16] Melrose R.B., Growth estimates for the poles in potential scattering, unpublished, 1984.

[17] Petkov V., Zworski M., Semi-classical estimates on the scattering determinant, Ann. Henri Poincaré 2 (2001), no. 4, 675-711.

[18] Ransford T., Potential theory in the complex plane, London Mathematical Society Student Texts, 28, Cambridge University Press, Cambridge, 1995.

[19] Regge T., Analytic properties of the scattering matrix, Il Nuovo Cimento 8 (1958), no. 10, 671-679.

[20] Sá Barreto A., Remarks on the distribution of resonances in odd dimensional Euclidean scattering, Asymptot. Anal. 27 (2001), no. 2, 161-170.

[21] Sá Barreto A., Zworski M., Existence of resonances in potential scattering, Comm. Pure Appl. Math. 49 (1996), no. 12, 1271-1280.

[22] Simon B., Resonances in one dimension and Fredholm determinants, J. Funct. Anal. 178 (2000), no. 2, 396-420.

[23] Sjöstrand J., Weyl law for semi-classical resonances with randomly perturbed potentials, Mém. Soc. Math. Fr. (N.S.) No. 136 (2014).

[24] Sjöstrand J., Zworski M., Complex scaling and the distribution of scattering poles, J. Amer. Math. Soc. 4 (1991), 729-769. 
[25] Sjöstrand J., Zworski M., Distribution of scattering poles near the real axis, Comm. Partial Differential Equations 17 (1992), no. 5-6, 1021-1035.

[26] Stefanov P., Sharp upper bounds on the number of resonances near the real axis for trapping systems, Amer. J. Math. 125 (2003), no. 1, 183-224.

[27] Stefanov P., Sharp upper bounds on the number of scattering poles, J. Funct. Anal. 231 (2006), 111-142.

[28] Vodev G., Sharp bounds on the number of scattering poles for perturbations of the Laplacian, Comm. Math. Phys. 146 (1992), no. 1, 205-216.

[29] Vodev G., Resonances in the Euclidean scattering, Cubo Matemática Educacional 3 no. 1 (2001), 317-360.

[30] Zerzeri M., Majoration du nombre de résonances près de l'axe réel pour une perturbation abstraite à support compact, du laplacien, Comm. Partial Differential Equations 26 (2001), no. 11-12, 21212188.

[31] Zworski M., Distribution of poles for scattering on the real line, J. Funct. Anal. 73 (1987), 277-296.

[32] Zworski M., Sharp polynomial bounds on the number of scattering poles of radial potentials, J. Funct. Anal. 82 (1989), 370-403.

[33] Zworski M., Sharp polynomial bounds on the number of scattering poles, Duke Math. J. 59 (1989), 311-323.

[34] Zworski M., Counting scattering poles. In: Spectral and scattering theory (Sanda, 1992), 301-331, Lecture Notes in Pure and Appl. Math., 161, Dekker, New York, 1994.

[35] Zworski M., Quantum resonances and partial differential equations, Proceedings of the International Congress of Mathematicians, Vol. III (Beijing, 2002), 243-252, Higher Ed. Press, Beijing, 2002.

Department of Mathematics, National University of Singapore, 10 Lower Kent Ridge Road, SINGAPORE 119076. http://www. math.nus. edu.sg/ matdtc

E-mail address: matdtc@nus.edu.sg

Université de Lille 1, Laboratoire De mathématiques Paul Painlevé, CNRS U.M.R. 8524, 59655

VILLENEUVE D’AsCQ CEDEX, FRANCE. http://www.math.univ-lille1.fr/ vnguyen

E-mail address: Viet-Anh. Nguyen@math. univ-lille1.fr 